\title{
Knowledge, Attitude, and Practice of Medication Abortion and associated factors among reproductive age women in selected sexual and reproductive Health clinics of Addis Ababa, Ethiopia, 2018: Cross sectional study
}

Addisu Yeshambel Wassie ( $\sim$ addisyes3@gmail.com )

Wolaita Sodo University https://orcid.org/0000-0002-6136-8998

Semarya Berhe Lemlem

Addis Ababa University

Abdisa Boka

Addis Ababa Fistula Hospital

Kelemu Abebe Gelaw

Wolaita Sodo University

Research article

Keywords: knowledge, attitude, practice, medication abortion, reproductive age women

Posted Date: July 8th, 2019

DOI: https://doi.org/10.21203/rs.2.10870/v1

License: (c) (i) This work is licensed under a Creative Commons Attribution 4.0 International License.

Read Full License 


\section{Abstract}

Background Abortion rates following unintended pregnancies is increasing in developing countries and unsafe abortion is commonly neglected reproductive health care problem. In Ethiopia, the number of women receiving treatment for complications from unsafe abortion nearly doubled from 2008-2014. Medication abortion is one of the safest abortion interventions. Adequate level of knowledge and attitude of reproductive age woman on medication abortion contributes to prevention of unsafe abortion. Objective Knowledge, attitude and practice of medication abortion and associated factors among reproductive age women in selected SRH clinics of Addis Ababa, Ethiopia, 2018. Method A crosssectional quantitative study design was conducted to collect data from study participants in sexual and reproductive health clinics of Addis Ababa from February-March 30, 2018. Study participants were selected through systematic random sampling. The data was coded and entered into Epi data version 4.2 and SPSS version 23 was used for analysis. Bivariate and multivariate analysis with $95 \% \mathrm{Cl}$ was employed. Variables found to have a P-value $<0.2$ in the binary logistic regression were entered into multivariate analysis and strength of association was declared at $P$ value $<0.05$. Results The overall knowledge, attitude and practice of medication abortion were $72.1 \%, 44.2 \%$ and $33 \%$ respectively. Majority (36.5\%) of the study participants would advise someone with unwanted pregnancy to undergo an abortion. Participants level of education (2ndry education((AOR=3.54, 95\% $\mathrm{Cl}[1.02,12.26])$, college/university (AOR=3.49, 95\% $\mathrm{Cl}[1.02,11.92])$ ), father education (up to 2 ndry education( $A O R=2.03$, $95 \% \mathrm{Cl}[1.01,4.10]$, college/university(AOR=2.80, 95\% CI[1.42, 5.52]), income(AOR=2.19, 95\% CI[1.02, 4.75]) and history of unwanted pregnancy $(A O R=11.7,95 \% \mathrm{CI}(1.11,12.46])$ were some of the factors associated with medication abortion. Conclusion and recommendations this study provided that women were relatively knowledgeable but their attitude and practice on medication abortion is low. Low attitude and practice of medication abortion calls health care providers giving due attention on awareness creation and strengthened action to provide quality of maternal care for childbearing mothers towards medication abortion and unsafe abortion in particular.

\section{Plain English Summery}

Unwanted pregnancies and unsafe abortion are commonly neglected reproductive health care problems in developing countries and pose major health risks to women in the reproductive age group. In many cases women with unintended pregnancy may try to end their pregnancies by themselves with the assistance of untrained personnel. It is reported that regardless of the availability of different medication abortion technologies, the problem of unwanted pregnancies is very big worldwide. Maternal morbidity and mortality as a result of unsafe abortion is perhaps one of the least discussed health problems in the Ethiopia. Thus, Problems like lack of women's awareness and perception over sexual and reproductive matters, incorrect or inconsistent use of contraceptive methods, and economic conditions, legal restrictions are not easily resolved and may lead to unintended pregnancies and lead to a profound impact on women's recourse to abortion and especially unsafe abortion. Access to medication abortion did not got enfaces because of different factors like law restrictions, Social, religious, cultural 
impediments, lack awareness, and maternal perception towards abortion facilitate delays in seeking medication abortion. Thus, when women in reproductive age group faces unwanted pregnancy, they may seek unsafe abortions like self-induction using different traditional abortifacient, may find traditional abortion providers regardless of the availability of abortion law and Unsafe abortions following unintended pregnancy become one of a major public health and human rights issue in the today's world. Therefore, the purpose of this study was to assess the knowledge, attitude and practice of medication abortion and associated factors among reproductive age women in selected sexual and reproductive clinics of Addis Ababa, Ethiopia. The study was conducted in SRH clinics in Addis Ababa, Ethiopia from February 30-March 30, 2018. Simple random sampling technique was used to select clinics and study participants were selected by using systematic random sampling technique. Data were collected by face to face interview technique using a structured questionnaire. Strength of association was determined using multivariable logistic regression model. Sexual and reproductive health clinics should take broad actions to empower women in every aspect of their lives especially in control of their reproductive lives.

\section{Background}

Abortion is termination of pregnancy (spontaneous, therapeutic or induced) before the fetus has become viable outside the uterus or before the fetus is capable to have a life outside of the womb(1). Medical abortion is the commonly performed safe abortion technology which uses medications in place of traditional surgical interventions for terminating an early unintended pregnancy. It is convenient, noninvasive and effective method with mortality rates for mifepristone-misoprostol combination regimens that are comparable to spontaneous abortion and success rates of $95-98 \%$ (2). Changing maternal mortality will be achievable if unsafe abortion will be replaced by medical abortion since thousands of lives could be saved each year by implementing medication abortion(3).

Maternal death and morbidity as a result of unsafe abortion is perhaps one of the least discussed health problems in the world(4). The study done by WHO and Guttmacher institute in 2010-2014, an estimated 56 million abortions occurred every year worldwide, of this 25 million (45\%) are unsafe abortions. The majority of unsafe abortions(97\%), occurred in developing countries in Africa, Asia and Latin America( 5). This number represents an increase from 50 million annually during 1990-1994, mainly because of population growth(6).

Ethiopia has a high maternal mortality ratio, 412 deaths per 100,000 live births for the period 2009-2016( 7) and an estimated 1.9 million (38\%) Ethiopian women have unintended of 4.9 million (62\%) total pregnancies and 620,300 induced abortions (13\%) were performed in 2014( 8, 9). From 2008 up to 2014, the proportion of abortions in health facilities were raised from $27 \%$ to $53 \%$, whereas, around 294,100 abortions occurred outside of health facilities in 2014(8). The number of women receiving treatment for complications from induced abortion nearly doubled which is 52,600 to $103,600(9,10)$.

Medication abortion especially the combination of mifepristone and misoprostol has currently come into focus as best alternative to manual vacuum aspiration in the world to terminate un intended pregnancy( 
11). A prospective study which was conducted in two hospitals and two clinics in northwest Ethiopia from March 2009 to November 2009 on client preferences and acceptability for medical abortion and manual vacuum aspiration as early pregnancy termination method suggested that participants undergoing termination of their pregnancies by using medication were reported greater satisfaction and more likely choose the same technologies again than were participants undergoing manual vacuum aspiration $(91.2 \%$ vs. $82.4 \% ; \mathrm{P}<.001)(12)$.

However, in Ethiopia, barriers and challenges to accessing medication abortion exist at multiple levels which may deny women's from using safe medication abortion technologies and exposing them to unintended births or to unsafe abortion and its distressing consequences of death and disability (13). Access to medication abortion did not got much enfaces because of different factors like law constraints, Social, religious, cultural impediments, lack awareness, and maternal perception towards abortion facilitate delays in seeking medication abortion. Thus, when women in reproductive age group faces unwanted pregnancy, they may seek unsafe abortions like self-induction using different traditional abortifacient, may find traditional abortion providers regardless of the availability of safe abortion techniques and Unsafe abortions following unintended pregnancy become one of a major public health and human rights issue in the today's world $(13,14)$.

Maternal morbidity and mortality due to unsafe abortion continue to occur regardless of major advances in the medical profession, especially in terms of the availability of safe and effective medication abortion technologies and skills for termination. The combination of mifepristone and misoprostol has been shown by various studies to be safe, effective and well-established method for termination of pregnancy. However, many women used less recognized and less effective abortive drugs( 11).

Even though many studies were conducted on abortion in Ethiopia, there seems to be a gap in evidence synthesis for women's knowledge, attitude and practice of medication abortion and as far as my knowledge is concerned, no facility based studies were conducted on knowledge, attitude, and practice of medication abortion among reproductive age women in Ethiopia. So to be widely used, medication abortion should be perceived and accepted by the women. Therefore, the purpose of this study was to assess the knowledge, attitude and practice of medication abortion and associated factors among reproductive age women in selected sexual and reproductive clinics of Addis Ababa, Ethiopia.

\section{Methods}

\section{Study area and setting}

The study was conducted in Addis Ababa, the capital of Ethiopia. Addis Ababa is set up in to ten subcities. According to the 2007 population and housing census, the city has a total population of 2,739,551 (3.7\%) and annual growth rate of $2.1 \%$ between 1994 -2007. Out of these, female population accounted for 1,434,164 (52\%). Women of reproductive age group among the total population are $947,855(15)$. According to the report from Addis Ababa FMHACA and Ethiopian $\mathrm{MOH}$, in the city, there are thirteen 
governmental hospitals, thirteen private hospitals, two NGO hospitals, twenty three governmental health centres, nine governmental clinics, one private health centre, three hundred seventy six private clinics, and seven SRH-clinics( 16).

\section{Study design and period}

Facility based cross-sectional quantitative study design was employed from February 30-March 30, 2018.

\section{Source population}

All reproductive age women attending in sexual and reproductive health clinics of Addis Ababa, Ethiopia, in 2018.

\section{Study population}

All reproductive age women attending in selected SRH clinics of Addis Ababa, Ethiopia in 2018.

\section{Inclusion criteria}

All reproductive age women coming for service in selected SRH clinics during data collection period were included.

\section{Exclusion criteria}

Reproductive age women coming for the service but involuntary, severely ill and whose age less than 18 years were excluded from the sample.

\section{Sample size determination}

The sample size required for study was calculated based on a single population proportion formula as follows.

[Due to technical limitations the formula could not be inserted here. If can be found in the supplemental file "Formulas"]

Where: $\mathrm{n}=$ sample size, $\mathrm{Z}=$ standard normal distribution corresponding to significance level at $\mathrm{a}=0.05, \mathrm{P}$ $=$ is anticipated proportion of women's knowledge, attitude and practice of medication abortion and it is assumed to be $50 \%$ taken to increase the sample size since there is no facility based study done on the target groups taken, $d=$ margin of error assumed to be $5 \%$ 
[Due to technical limitations the formula could not be inserted here. If can be found in the supplemental file "Formulas"]

By considering $10 \%$ non-response rate, the total sample size was $384+39=423$

\section{Sampling procedure}

Clinics were selected by using simple random sampling and systematic random sampling technique was employed to select subjects. First the total sample size was distributed to each selected SRH clinics proportionally to make it representative. Then exit interview was employed (participants were selected using systematic random sampling technique or every $\mathrm{k}^{\text {th }}$ exit ( $\mathrm{k}^{\text {th }}$ value))

\section{Dependent variable}

- Knowledge on medication abortion

- Attitude towards medication abortion

- Practices of medication abortion

\section{Independent variable}

- Socio-demographic \& economic variables( age, educational status, residence, religion, place of birth, marital-status, occupation, income, parental educational status)

- Obstetric variables(parity) and

- RH service variables(contraceptive history, Friends or relative/family who has had abortion, history of unwanted pregnancy)

\section{Operational definition}

- Medication abortion: is a method of termination of early first trimester pregnancy (meaning by using mifepristone, misoprostol or both). It can be initiated up to 7-9 weeks (49-63 days) of gestation (via menstrual dating).

- Knowledge: what a woman knows about medication abortion (Meaning of MA, place where it can be provided, which drugs can be used for medication abortion and when it can be used (gestational age)).

- Low knowledge: If the participant score's less than or equal to two on the knowledge part questions

- Satisfactory knowledge: A score of three on the knowledge part of the assessment 
- High knowledge: A score of greater than or equal to four on the knowledge part of the assessment(17).

- Attitude: the tendency to respond in a favourable or unfavourable way towards abortion, medication abortion and other abortion related issues such as advising/recommending colleague to have abortion or for oneself in case of unplanned pregnancy, which type of abortion is desirable.

- Positive attitude: participants who scored the mean and above value from the provided attitude related questions about the medication abortion.

- Negative attitude: participants who scored below the mean value of the provided attitude questions about the medication abortion

- Practice: is the explicit health behaviour, practice of a woman related to medication abortion or those who practiced medication abortion at least once in past.

\section{Methods of data collection and tools}

Structured and pre-tested questionnaire consisting of items with pre-coded response categories was used to collect data from the study participants. The questionnaire was adapted \& modified by reviewing a literature( 18). The tool was $1^{\text {st }}$ designed in English and translated in to local Amharic language and then translated back to English by a professional language translators/editors to check for its consistency.

\section{Data collection procedure and quality control}

Data was collected by face to face interview using structured questionnaires. Five BSc nurses and three BSc midwifes were selected to collect the data and two BSc midwives were selected for supervision. Before starting the actual data collection to assure the data quality, high emphasis was given to designing data collection instrument, first the questionnaire was pre-tested on $10 \%$ of sample size or 43 mothers on Gotera SRH clinics. After pre-testing the questionnaire, further adjustments to the data collection tool was made to improve clarity, understand-ability, and simplicity of the messages. All of the questionnaires were checked for completeness and accuracy before, during and after the period of data collection. Throughout the course of the data collection, interviewers were supervised, regular meetings were held between the data collectors and the principal investigator together in which problematic issues arising from interviews during the data collection were discussed. The collected data was again reviewed and checked for completeness before data entry. Data entry format template was prepared and programmed by principal investigator.

\section{Data analysis and processing}

First the collected data was checked manually for completion and any incomplete or misfiled questions. Then the data was cleaned and stored for consistency and entered in to Epi Data version 4.2, and then it 
was exported to statistical package for social sciences (SPSS) version 23.0 software for analysis.

Descriptive statistics like frequency, proportion, mean, and standard deviation were computed to describe study variables in relation to the population. Logistic regression (bivariate and multivariate) was used to determine the effect of independent variables on the outcome variables. The strength of association was declared at $P$ value $<0.05$. Variables found to have a $P$-value $<0.2$ in the binary logistic regression was entered/exported into multivariate analysis to identify their independent effects and the final results were presented as odds ratio (OR). Finally, results were compiled and presented using texts, tables, graphs.

\section{Ethical consideration}

Ethical clearance and approval were obtained from Addis Ababa University, College of Health Sciences, and school of nursing and midwifery ethical review committee. Then, letter from the Research Ethics Committee was submitted to Addis Ababa Health Bureau and research committee of SRH clinics then letters from research committee of SRH clinics was submitted to the selected SRH clinics. After explaining the objectives of the study in detail, informed written consent was taken from all study participants. All the participants were reassured of the anonymity, and as personal identifiers were not used. Then, after obtaining informed consent from every participant, the data collectors were continued the job by giving due respect to the norms, values, beliefs, culture, and ensured the confidentiality of the data.

\section{Dissemination of the result}

The results of this finding will be disseminated or communicated to Addis Ababa

University, College of Health Science, School of Nursing and Midwifery. It will also disseminated to Addis Ababa Health bureau and other concerned bodies through reports and publication on an appropriate journal. Efforts made to present the results on scientific conferences and publications will be considered.

\section{Results}

\section{Socio-Demographic Characteristics of Study Population}

From the total of 423 mothers who were invited for interview, 412 filled the questionnaires completely resulting in a response rate of $(97.4 \%)$. Mean age of the respondents was 25.0 ( $S D \pm 5.11)$ years with a minimum and maximum age of 18 and 43 respectively. Majority of the respondents $133(32.3 \%)$ fall in between 24-27 years age group. Out of the total respondents, 294 (71.4\%) of them were born in Addis Ababa and majority $238(57.8 \%)$ of the study participants were orthodox religion followers. From the total participants; 145 (35.2\%) had attended up to secondary education followed by college/university 139 (33.7\%). Regarding the marital status of the respondents 132 (32\%) were married, while the majority 
which account 178 (43.2\%) were single without relationship. Almost all 386 (93.7\%) were live in Addis Ababa where $88(21.4 \%)$ were private employers and out of the total respondents $138(33.5 \%)$ of them had a monthly income of $<500$ Ethiopian birr (Table 1 ).

\subsection{Socio-demographic background of their parents}

Majority 199 (48.3\%) of the study participants had illiterate mother, 114 (27.7\%) with primary education, $54(13.1 \%)$ had secondary education and $45(10.9 \%)$ had some forms of college education. On the other hand $180(43.7 \%)$ of their fathers' were reported to be

illiterate, whereas, $87(21.1 \%)$, had some forms of college education. Regarding the medical related profession in their family, $130(31.6 \%)$ of the study participants were reported to have medical related profession in their family (Table 2 ).

\subsection{The general knowledge of participants' on abortion}

Majority or more than two thirds of the participants responded as giving birth 281 (68.2\%) and 232 (56.2\%) said abortion related to their possible outcomes of pregnancy, Majority $325(78.9 \%)$ of participants were informed of abortion. When asked about the methods of termination/performing abortion participants replied that; by medication /drugs 260 (80\%), by Surgical procedures $50(15.4 \%)$, and by traditional practitioners $29(8.9 \%)$. More than half $240(58.3 \%)$ of the participants were awared of safe abortion (Table S1).

Regarding to information source, more than one third of respondents $180(55.4 \%)$ mentioned friends as followed by health facility 87 (26.8\%), and media (television, radio and newspapers) 59 (18.2\%) respectively (Fig 2).

\subsection{General Knowledge and attitude of participants on abortion law}

Majority of the respondents, $168(40.8 \%)$ reported as abortion is legally allowed in Ethiopia while 135 $(32.8 \%)$ believed that abortion is not allowed in the country. From those study participants who believe abortion is legally allowed in the country $(n=168)$, majority of the participants mentioned rape or incest 131 (78\%) followed woman or fetus lives are threatened $107(63.6 \%)$ as the main reasons in which unintended pregnancy will be happened(figure 1). More than half of the respondents, 247 (60\%) support government allowing abortion in Ethiopia. From those who support government to allow abortion $(n=247)$, majority,167 (67.7\%) were mentioned to prevent the death of women due to unsafe abortion followed by to prevent unsafe abortion and it is a human right which is 103 (41.7\%), 64 (25.9\%) respectively as a reason abortion to be allowed. Similarly, from the total $(n=165)$, who do not support the 
government to allow abortion, majority, 122 (73.9\%), reported not allowed in their religion mentioned the main reason followed by as it encourages many women to have unplanned pregnancy $93(56.4 \%)$, it is crime 45 (27.3\%), and it will cause risk on health of women 45 (27.3\%)(Table S2).

\subsection{Knowledge regarding Medication Abortion}

Three fourth $312(75.7 \%)$ reported that as they knew the definition of MA. from those who defines MA, 221 (70.8\%), 51 (16.3\%), $33(10.6 \%)$ and $7(2.2 \%))$ reported as: MA means; by termination of unintended pregnancy by using abortion drugs, using any other medication, by inserting objects through the birth canal and termination by drinking chemicals respectively. Of 312 respondents that defines MA, 263 $(84.3 \%)$ also reported as they knew where medication abortion service will be given. The majority of the respondents 199 (63.8\%) do not have knowledge on medication abortion drugs. However, some of the respondents mentioned drugs that can be used in case of MA like Misoprostol 86 (27.6\%), Mifepristone 67 (21.5\%), Amoxicillin 6 (1.9\%), Ampicillin 7(2.2\%), Methotrexate 8 (2.6\%), Gemprost 5 (1.6\%) and Safe-T 5 (1.6\%). Majority, 116 (37.2\%) of the participants who knew about MA, did not understand when MA will be provided/taken. But 111 (35.6\%) of respondents reported as it will be taken at the gestational age less than or equal to 9 weeks followed by gestational age less than or equal 63 days 74 (23.7\%), and few of them $5(1.6 \%)$ responded MA to be performed at any gestational age (Table S3).

As it has been shown in (Fig 2), out of 312 respondents who knew about MA, 148 (47.4\%) said that they got the information from friends followed by health facility/hospital, $133(42.6 \%)$. From those who claimed to know where MA will be performed, majority, 181(68.8\%) answered health centre followed by hospital 103 (39.2\%) (Fig 3). Though the majority of the respondent knew what MA means and when knowledge score was done using knowledge questions, it was found that most of the study participants have high knowledge on MA 159 (38.6\%) followed by satisfactory knowledge 138 (33.5\%)(Fig S1).

\subsection{Attitude towards medication abortion}

From the total respondents, 150 (36.4) reported that as they would advise or encourage someone with unwanted pregnancies to undergo abortion. The most preferred method that they advise for someone was abortion by medication 131 (87.3\%) followed by surgical procedures $18(12 \%)$ and by traditional practitioner $10(6.7 \%)$. More than half of the respondents, $230(55.8 \%)$ believed that if they had an unplanned pregnancy, they would consider abortion to terminate it and the reasons that they believe were: inability to raise their child 139 (60.7\%) followed by it will affect my education 69 (44.5\%). Abortion by medication /drugs from health facility was the preferred way of abortion 195 (84.8\%). Of the respondents who didn't consider abortion $(n=182)$ if they had an unplanned pregnancy, majority $146(80.2 \%)$ reported that as their religion cannot allow abortion followed by I don't want to kill my own baby 94 (51.6\%) (Table S4). 


\subsection{Sexual and medication abortion practice}

Nearly half 201 (48.8\%) of the respondents know a friend/neighbour who had terminated pregnancies and almost all 409 (99.3\%) of the respondents had sexual intercourse experience, from those who had sexual intercourse before, $193(46.8 \%)$ of them had history of unwanted pregnancy. Out of the total study participants, $206(50 \%)$ had a history of termination of pregnancy and majority of the respondents report as: I know, but I did not use contraceptives 97 (47.1\%) followed by I know and used contraceptives but I faced pregnancy $53(25.1 \%)$ as a reason for termination. From those $(n=206)$ respondents, majority 136 (66.2) used medication/drugs for termination followed by abortion by surgical procedure 35 (17.2\%). Being easy to take $81(39.71 \%)$ is the most given reason for MA preference (Table S5).

\subsection{Determinants of knowledge, attitude and practice towards medication abortion}

\subsubsection{Socio-economic variables, parent related factors, and reproductive health service related factors associated with medication abortion knowledge}

Binary Logistic regression was performed to assess the association of each independent variable with medication abortion knowledge. The factors that showed a p-value of less than 0.2 were added to the multivariate regression model. The result revealed that on the bivariate analysis: Age group, place of birth, their way of living, their father's educational level, medical related profession in their family and history of unwanted pregnancy were significantly associated with medication abortion knowledge. In multivariate logistic regression, fathers' levels of education and their way of living were significantly associated with medication abortion knowledge at P-value of $<0.05$ (table 8). Respondents who have a father learnt up to secondary education were 2.03 times more knowledgeable on the MA than with a father who is illiterate (AOR $=2.03,95 \% \mathrm{Cl}[1.01,4.10]$. Similarly, those respondents whose father learnt some forms of college or university education were 2.80 times more likely knowledgeable on MA than respondents with a father who is illiterate(AOR=2.80,95\% $\mathrm{Cl}[1.42,5.52]$. In addition to this, those respondents living with their relatives (2.34 times), with their boyfriends ( 6.08 times), and with their friends ( 5.35 times) were more likely knowledge on MA compared with those living with their family (AOR=2.34, 95\% $\mathrm{Cl}[1.05,5.25]$ ), $(A O R=6.08,95 \% \mathrm{Cl}[2.41,15.33])$, and $(\mathrm{AOR}=5.35,95 \% \mathrm{CI}[2.18,13.16])$ respectively (Table S6).

\subsubsection{Socio-economic variables, parent related factors, obstetric factor and reproductive health service related factors associated with respondent's attitude towards medication abortion}


Bivariate and multivariate logistic regression analysis was employed to calculate odds ratios and corresponding $95 \%$ confidence intervals for the predictors of MA attitude. Variables which were significant in the bivariate analysis of $p$-value $<0.2$ were place of birth, religion, marital status, income, their fathers' level of education, and medical related profession in the family. As a result, medical related profession in the family was remained in the multivariate logistic regression due to its statistical significance. Hence, the result of multivariate analysis showed that study participants who had a medical related profession in their family had positive attitude towards MA compared with those who had no medical related profession in their family(AOR=1.56, 95\% Cl [1.01, 2.42]] (Table S7).

\subsubsection{Socio-economic variables, parent related factors, and reproductive health service related factors associated with respondent's practice towards medication abortion}

As shown in table 10, variables which show significant association in the bivariate analysis of $p<0.2$ (respondents' level of education, income, knowing a family member/friend/neighbour who had terminated a pregnancy, respondents who had history of unwanted pregnancy and respondents who know contraceptives) were entered into multivariate analysis. The participants' level of education, income and respondents who had history of unwanted pregnancy were significantly associated with the respondents MA practice (table 10). When we compare MA practice among educated and illiterate respondents, respondents who had learnt secondary education (3.54 times) and college/university (3.49 times) were more likely to practice MA than illiterates(AOR=3.54, 95\% $\mathrm{Cl}[1.02,12.26])$, (AOR=3.49, 95\% $\mathrm{CI}[1.02,11.92])$ respectively. Similarly respondents who had history of unwanted pregnancy were 11.7 times more likely practice to MA than those respondents who had no history of unwanted pregnancy $(A O R=11.7,95 \% \mathrm{Cl}(1.11,12.46])$ In addition, respondents who get a monthly salary of greater than 1000 ETB were 2.19 times more likely to practice MA than those who get monthly salary less than 500 $\operatorname{ETB}(\mathrm{AOR}=2.19,95 \% \mathrm{Cl}[1.02,4.75])($ Table 58$)$.

\section{Discussion}

The study has investigated the knowledge, attitude and practices of MA and associated factors among reproductive age women in selected SRH clinics of Addis Ababa, Ethiopia. Around 325(78.9\%) of the respondents have heard about abortion. Friends (55.4\%) were the main source of information followed by from health facility $(18.2 \%)$ and media (26.8\%). This finding was lower than the study conducted in evangelical women in Addis Ababa where $93.5 \%$ have heard about abortion where media accounts (28.1\%)( 19), and social science students in Addis Ababa University which showed that the entire population $(100 \%)$ have heard about abortion and media (82.6\%) was the main source of information about abortion followed by friends $(70.0 \%)(18)$. This difference might be due to study group differences or the negligence/decreased emphasises of media (Radio, television, newspaper, and the like) and health care providers on information dissemination regarding reproductive issues. 
According to this study, more than half of the respondents have heard about safe abortion (58.3\%) and unsafe abortion (57.3\%) and this study was higher than the descriptive study carried out on 50 reproductive age women in Lekhnath, kaski, Nepal, on the assessment of the level of knowledge regarding safe abortion which showed that only $40 \%$ of the respondents knew safe abortion(20). This might be due to study time difference or due to small sample size carried out in Nepal.

About 168 (48.8\%) of the study participants said abortion is liberal and allowed in Ethiopia under some conditions, however, 135 (32.8\%) said abortion is not legally allowed in Ethiopia, and 109 (26.5\%) said they don't know whether it is allowed or not. Among those respondents who said abortion is legally allowed in Ethiopia ( $n=168$ ), most of the respondents mentioned that abortion is allowed only for the conditions like; when the pregnancy is resulted from rape or incest $(131(78.0 \%))$, when the woman or fetus lives are threatened $(107(63.7 \%))$, when the fetus has severe abnormalities (90 (53.6)) and some of the study participants $(29(17.3 \%)$ responded on request for everyone. This finding was in line with the study done in Yirga cheffe town SNNPR on knowledge and attitude of women of childbearing age towards legalization of abortion in Ethiopia, which showed that $48.9 \%$ were aware of legalization of abortion law(21). This might be due to the same sample size used in both studies or the contribution of the health care providers on the dissemination of information towards legalization. In contrast to this, the finding was slightly lower than the study done in Nepal(20), the study conducted in Addis Ababa among evangelical woman which showed that abortion is allowed only for the conditions like; when the pregnancy is resulted from rape or incest $(11.5 \%)$ ), when the woman lives are threatened $(37.20 \%)$ ), every women's request(8.33\%)(19) and the study done in three lower districts in Bihar Dar, north west Ethiopia, which showed that 512 (66\%) were aware of the legal status of the Ethiopian abortion law(22). This difference might be due the large sample size used in the study conducted in Bihar Dar, small sample size used in study conducted in Addis Ababa and it may be due to the health policy, program and strategy differences and technological differences with Nepal.

Regarding to MA, more than three-fourth of respondents 312 (75.7\%) have aware of what MA means, but $88(21.4 \%)$ of them do not know about it. Among the 312 respondents who claimed to know about MA, majority $221(70.8 \%)$ has reported as MA means: abortion using abortion pill/drugs. When the knowledge score was done $38.6 \%, 33.5 \%$ of the respondents who knows MA had high and satisfactory knowledge respectively, and the reaming $27.9 \%$ of them had low knowledge towards MA. This study was higher than the study done in Brazil on medication abortion knowledge showed that the percentage of the respondents who had heard about medication drugs as a means to induce abortion was $72 \%$ though only less than $3 \%$ had satisfactory general knowledge( 17$)$. The difference in the result might be due to socio cultural and socio economical or health police, implementation program difference.

Accordingly the finding of this study revealed that, more than two thirds of the respondents 199 (63.8\%) do not know which drugs are used in case of MA but some of the respondents who had reported to know MA reported as Misoprostol 86 (27.6\%), mifepristone 67 (21.5\%), Amoxicillin 6 (1.9\%), Ampicillin 7 (2.2\%), Methotrexate 8 (2.6\%), Gemprost 5 (1.6\%) and Safe-T 5 (1.6\%). This finding was higher than the study done on MA seekers in south eastern Nigeria, which showed that only $3 \%, 2 \%$ and $0 \%$ knew misoprostol, 
mifepristone and methotrexate( 20). These differences might be due to small sample size used in previous study or it might be due poor access of reproductive services, restrictive abortion laws or difference in health police, implementation program and study time differences. Similarly the findings of this study were higher than the study done in reproductive age group Evangelical women residing in Addis Ababa where 20.8\% knew specific abortifacient medicines like Mifepristone and Misoprostol, $13.20 \%$ knew about antibiotics and while $13.4 \%$ replied that they do not know any method and study done in social students in Addis Ababa which showed that Misoprostol 3 (1.9\%), Mifepristone 2 (1.2\%), Methotrexate $2(1.2 \%)$, and Safe-T $3(1.9 \%)$ (18). These discrepancies might be due to the difference in study setting and subject or it may be sampling technique or sample size used or might be due to the fact that students can access better information than woman.

In this study most of the respondents who claimed to know MA, reported as when medication abortion was preferable. From those respondents 111 (35.6\%) said, when the pregnancy is less or equal to 9 weeks, $74(23.7 \%)$ said when the pregnancy was less or equal to 63 days and few $5(1.6 \%)$ said at any gestational age. However, majority $116(37.2 \%)$ did not know when medication abortion will be performed. This finding was slightly lower than the study done in India to assess the knowledge, attitude and practice of MA which showed that majority $53.3 \%$ taken abortifacient at less than 12 weeks gestation and $26.6 \%$ of them did not know were MA will be given( 23 ). These might be due to the educational level, technological differences or may be due to better health policy and program implementation in India.

On the other category, more than two thirds of the respondents 247 (60\%) were reported to support the idea that government should allow abortion in Ethiopia. From the respondents $(n=247)$, majority 167 (67.6\%) reported to support the idea to prevent death of women due to unsafe abortion, $41.7 \%$ said to prevent unsafe abortion as a result of unwanted pregnancy and some $(25.9 \%)$ reported as it was a human right. And from the respondents who do not support the government to allow abortion, majority $122(73.9 \%)$ claimed that their religion did not allow them to abort, $56.3 \%$ of them reported that as it will encourage many women to have unplanned pregnancy and $27.3 \%$ of them said as it is a crime in the country. The study was consistent with the study done in India on the assessment of attitude and experience of married women which showed that the majority of the study participants would seek MA to prevent unwanted pregnancy which will mostly lead to unsafe abortion. This might be due to the common understanding of the countries on the prevention of unwanted pregnancy which may mostly end up unsafely.

In this finding, $44.2 \%$ of the participants had positive attitude towards MA and majority $150(36.4 \%)$ of them reported to advise or encourage a colleague with unwanted pregnancies to undergo an abortion. Of those, $87.3 \%$ of them may advise their colleague to choose abortion with medication/drugs. On the other hand more than half, $55.9 \%$ of the respondents consider abortion as a means of solution when they face unwanted/unplanned pregnancy and most $60.7 \%$ claimed that as they can't raise the child, $44.5 \%$ of them reported that as it may affect their education and around $25.8 \%$ of them consider abortion due to fear of social stigma. The study was consistent with the study done in Guraghe zone, which showed that 
$40.8 \%$ of them had induced abortion as it may affect their education followed by $36.7 \%$ had due to unable to raise their children because of economic reasons(24). This might be due to the attitude of the respondents for pregnancy and socio economic similarities across the regions in country.

Regarding to medication abortion practice, only $33 \%$ practiced medication abortion from the total study participants. $46.8 \%$ of the respondents had history of unwanted pregnancy and $49.8 \%$ of them had history of termination of pregnancy. From those who had terminated their pregnancy, majority $66.7 \%$ used abortion pill/drugs for termination followed by abortion by surgical procedures $17.2 \%$ and by traditional Practitioners $16.2 \%$. Many of them claimed the reason for termination were they know, but negligent to use contraceptives(47.1\%), followed by lack of knowledge $(27.2 \%)$ and some of them were claimed that as they become pregnant as they were on contraceptives(25.7\%). This figure was higher than the study conducted in India which showed only $17.5 \%$ used MA (25) and the study was slightly higher than the study done in Nigeria, which showed $49 \%$ had previous termination and $48 \%$ had used drugs for termination of pregnancy (11). This inconsistency might be due to difference in health police, implementation program and small sample size used in the previous study.

In this study father's educational level were significantly associated with medication abortion knowledge which shows that respondents who have a father learnt secondary education were 2.03 times more knowledgeable on MA than with fathers who are illiterate. Similarly, those respondents whose father learnt college/university were 2.80 times more knowledgeable on MA than respondents whose father is illiterate. This finding was consistent with the study done in India(25), Addis Ababa( 18), which showed that respondents with fathers who are illiterate had low knowledge regarding MA compared to respondents having fathers who are educated (AOR=0.112 [0.013-0.969]). In addition to this, from this study 'participant's way of living' were significantly associated with MA abortion. Those respondents living with their relatives [AOR=2.34, $\mathrm{Cl}$ [1.05-5.25]], boyfriends [AOR= [6.08, $\mathrm{Cl}$ [2.41-15.33]] and with their friends $[A O R=5.35, \mathrm{Cl}[2.18-13.16]]$ had knowledge on MA compared with those living with their family respectively. This finding was comparable to the study conducted in Dar Es Salaam, Tanzania, on unwanted pregnancy and induced abortion among females, which showed that majority 17 (65.4\%) of the respondents living with their boyfriend and 3 (11.5) living with their friends had significant knowledge on abortion(4).

In addition to this, when we compare MA practice among educated and illiterate respondents, respondents who had secondary education (3.54 times) and college/university (3.49 times) were more likely to practice MA than illiterates. This finding was similar to the study done in India (25) and in Addis Ababa (19) which showed that, usage of MA drugs was significantly higher than in educated group. Similarly, respondents who had history of unwanted pregnancy were 11.7 times more likely to practice MA than those respondents who had no history of unwanted pregnancy. This finding was consistent with the study done on determinants of abortion practice in Addis Ababa, which showed that respondents with history of unwanted pregnancy $(p<0.000)$ had a strong association with their unwanted pregnancy $(26)$. In addition to the above factors, respondents who get a monthly salary of greater than 1000 ETB were 2.19 times more likely to practice MA than those who get less than 500ETB. This finding is comparable 
with the study conducted in evangelical woman residing in Addis Ababa(19) which showed that respondents' income level were strongly associated with abortion practices. This might be due the same socio-economic background of the country.

\section{Strengths of the study}

- Since it is primary data, those governmental and non-governmental organizations involved in maternal empowerment can utilize the findings of the study as information for intervention of programs and for further study.

\section{Study limitations}

Abortion is still a sensitive issue; some of the responses by the respondents might have been influenced by religious, cultural and personal beliefs. So that respondent may answer questions in a manner that would be viewed favourable by others (may under / inaccurately report their view towards medication abortion). Recall bias and white coat bias might not be minimized. Since the data is collected at a single point in time, temporal relationship could not be established.

\section{Conclusion}

Generally, from this study majority (78.9\%) of the study participants heard about abortion, only $75.7 \%$ had awareness on medication abortion and the major sources of information were friends and from a health facility. Nearly one-third of the respondents (27.9\%) were found to have low knowledge score on medication abortion. Medication abortion is acceptable by the study participants where one third of the respondents have positive attitude (44.2\%) towards abortion by advising or encouraging colleague $(36.2 \%)$ with an unwanted pregnancy to undergo an abortion where most of them would advise or encourage abortion by medication /drugs (Medication Abortion). Of all the respondents, 230 (55.8\%) would consider abortion to terminate if they have unplanned pregnancy and $33 \%$ of them had practiced medication abortion from the total participants. Respondents' level of education, father's educational level, their way of living, history of unwanted pregnancies, medical related profession in family and monthly income were identified as major determinants (predictors) of medication abortion knowledge, attitude and practice in this particular study.

\section{Recommendations}

Sexual and reproductive health (SRH) clinics: Should take broad actions to empower/encourage women in every aspect of their lives especially in control of their reproductive lives. They should help a woman to make: 
- Their own fertility decisions and have access to and know where to find medication abortion services in their communities.

- Permitting services to be visible, accessible and affordable for all women

- Helping health professionals feel protected in providing compassionate abortion services

- Allowing women to choose abortion methods, providers and when it will be taken

- Better enabling women to take legal action when their rights are violated

Media: Should better disseminate the conditions under which abortion is currently permitted, the availability of medication abortion, and where the woman's can access information regarding about medication abortion.

Health care providers: The Health workers, particularly health extensions should teach the community about the consequence of unsafe abortion and stigma.

Organizations including Ethiopian midwifes association (EMwA): Both governmental and nongovernmental organizations who were primarily involved in maternal health should address the issue of negative attitude to change the communities towards safe abortion services especially on medication abortion.

Policymakers and $\mathrm{MOH}$ : Should put restrictions on the over the counter sale of drugs that are used for medical abortion and provision should be made to make these drugs available only on authorised prescription from qualified personals.

Researchers: Similar studies should be undertaken in different parts of the country to see the trends, magnitude and the determinant factors of medication abortion.

\section{Aabbreviations}

EDHS-Ethiopian Demographic Health survey: FMHACA-Food, Medicine and Health administrative and control authority: MA-Medication abortion: $\mathrm{MCH}$ - Maternal and child Health: $\mathrm{MOH}-$ Ministry of Health: $\mathrm{RH}-$ Reproductive Health: SPSS- Statistical package for social sciences: STI- Sexually transmitted infections: $\mathrm{SRH}-\mathrm{Sexual}$ and reproductive Health: WHO- World Health organization

\section{Declaration}

\section{Ethics approval and consent to participant}

Ethical clearance and approval letter was obtained from Addis Ababa University. Personal patient information was not recorded, after finishing the data collection, the questionnaire was kept in appropriate place and the information was used for study purpose only. 


\section{Consent for publication}

Not applicable.

\section{Availability of data and material}

The data sets used and/or analysed during the current study are available from the corresponding author on reasonable request.

\section{Competing interests}

The Corresponding author declare that there were no competing interests

\section{Funding}

Addis Ababa University was involved for financial support for data collectors, and expenditure for translation and transcription of questionnaires and for hard copies.

\section{Authors-contributions}

AY was involved in the conception, design, analysis, interpretation, report, manuscript writing, design, analysis, interpretation and report writing. $\mathrm{SB}, \mathrm{AB}$ and $\mathrm{KA}$ were involved design, analysis and interpretation of the data. All authors read and approved the final manuscript.

\section{Acknowledgments}

We would like to express our deepest heartfelt thanks to Addis Ababa University for allowing the conduct of this study. Our especial thanks go to SRH clinic staffs for their support during the data collection process.

\section{Authors' information}

Addisu Yeshambel Wassie is Lecture at Wolayita Sodo University in the department of Midwifery, College of Health Science and Medicine, Wolaita Sodo University, Wolaita Sodo, Ethiopia.

Semarya Berhe Lemlem is Assistant professor in School of Nursing and Midwifery, College of Health Science, Addis Ababa University, Addis Ababa, Ethiopia. 
Abdisa Boka is Lecture in School of Nursing and Midwifery, College of Health Science, Addis Ababa University, Addis Ababa, Ethiopia.

Kelemu Abebe Gelaw is Lecturers in the department of Midwifery, College of Health Science and Medicine, Wolaita Sodo University, Wolaita Sodo, Ethiopia.

\section{Reference}

1. Organization WH. Safe and unsafe induced abortion: Global and regional levels in 2008 , and trends during 1995-2008. 2012.

2. Borkowski L, Strasser J, Allina A, Wood S. Medication Abortion: Overview of Research \& Policy in the United States-References by Topic Area. 2015.

3. Baggaley RF, Burgin J, Campbell OM. The potential of medical abortion to reduce maternal mortality in Africa: what benefits for Tanzania and Ethiopia? PLoS One. 2010;5(10):e13260.

4. Mamboleo N. Unwanted pregnancy and induced abortion among female youths: a case study of Temeke district: Muhimbili University of Health and Allied Sciences; 2012.

5. institute G. Induced Abortion Worldwide. september 2017.

6. Sedgh G, Henshaw S, Singh S, Åhman E, Shah IH. Induced abortion: estimated rates and trends worldwide. The Lancet. 2007;370(9595):1338-45.

7. Central Statistical Agency I. ETHIOPIA Demographic and Health Survey 2016. BMC public health. 2016;15(1):139.

8. Gebrehiwot Y, Fetters T, Gebreselassie H, Moore A, Hailemariam M, Dibaba Y, et al. Changes in morbidity and abortion care in Ethiopia after legal reform: national results from 2008 and 2014. International perspectives on sexual and reproductive health. 2016;42(3):121-30.

9. Singh S, Fetters T, Gebreselassie H, Abdella A, Gebrehiwot Y, Kumbi S, et al. The estimated incidence of induced abortion in Ethiopia, 2008. International perspectives on sexual and reproductive health. 2010:1625.

10. Moore AM, Gebrehiwot Y, Fetters T, Wado YD, Bankole A, Singh S, et al. The Estimated Incidence of Induced Abortion in Ethiopia, 2014: Changes in the Provision of Services Since 2008. International perspectives on sexual and reproductive health. 2016;42(3):111-20.

11. Adinma ED, Adinma JIB-D, Iwuoha C, Akiode A, Oji E, Okoh M. Knowledge and practices among medical abortion seekers in southeastern Nigeria. Southeast Asian Journal of Tropical Medicine and Public Health. 2012;43(2):471. 
12. Woldetsadik MA, Sendekie TY, White MT, Zegeye DT. Client preferences and acceptability for medical abortion and MVA as early pregnancy termination method in northwest Ethiopia. Reproductive health. 2011;8(1):19.

13. Assifi AR, Berger B, Tuncalp O, Khosla R, Ganatra B. Women's Awareness and Knowledge of Abortion Laws: A Systematic Review. PLoS One. 2016;11(3):e0152224.

14. Alemu FF. Minors' awareness about the new abortion law and access to safe abortion services in Ethiopia: the Case of Marie Stopes International Ethiopia Centers in Addis Ababa. Unpublished thesis, University of Amsterdam, Addis Ababa. 2010.

15. Samia Zekaria member and Secretary PCC. Population and Housing Census of Addis Ababa, Ethiopia. 2007.

16. Health FMo. health and health related indicators. 2007.

17. Fernandes KG, Camargo RPS, Duarte GA, Faúndes A, Sousa MH, Maia Filho NL, et al. Knowledge of medical abortion among Brazilian medical students. International Journal of Gynecology \& Obstetrics. 2012;118(S1).

18. Thomas G. Assessment of Knowledge, Attitude and Practices regarding Medication Abortion among Regular Undergraduate Female Students in College of Social Sciences, Addis Ababa University: Addis Ababa University Addis Ababa, Ethiopia; 2014.

19. Solomon H. KNOWLEDGE, ATTITUDE AND PRACTICE ASSESSMENT TOWARDS INDUCED ABORTION AMONG EVANGELICAL WOMEN RESIDING IN ADDIS ABABA. 2015.

20. -63.9Acharya A, Bhattarai S. Assessment of the level of knowledge regarding safe abortion among reproductive age group women, a short cross sectional study in lekhnath, kaski. Journal of Institute of Medicine. 2017;41(1).

21. Bitew S, Ketema S, Worku M, Hamu M, Loha E. Knowledge and attitude of women of childbearing age towards legalization of abortion, Ethiopia. Journal of Scientific \& Innovative Research. 2013;2(2):192-203.

22. Muzeyen R, Ayichiluhm M, Manyazewal T. Legal rights to safe abortion: knowledge and attitude of women in North-West Ethiopia toward the current Ethiopian abortion law. public health. 2017;148:129-36.

23. Namrata S, Sumitra Y. The Study of Knowledge, Attitude and Practice of Medical Abortion in Women at a Tertiary centre. Pain.57:27.14.

24. Tesfaye G, Hambisa MT, Semahegn A. Induced abortion and associated factors in health facilities of Guraghe Zone, southern Ethiopia. Journal of pregnancy. 2014;2014. 
25. Lakkawar N, Magon S, Alaganandam P. Attitude and experiences of young women towards medical abortion a hospital based study. Scholars Journal of Applied Medical Sciences. 2014;2(6B):2034-41.

26. Habte M. Determinants of abortion practice in Addis Ababa: MA thesis submitted to Population studies, Addis Ababa university; 2010.

\section{Tables}

Due to technical limitations the tables could not be inserted here. They can be found in the supplemental files]

\section{Supplemental Figure Legend}

Figure S1: Knowledge score for medication abortion among reproductive woman in selected sexual and reproductive health clinics of Addis Ababa, February 30-March 30, 2018.

\section{Figures}




\section{Conditions where the law permits terminating pregnancy $(n=168)$}

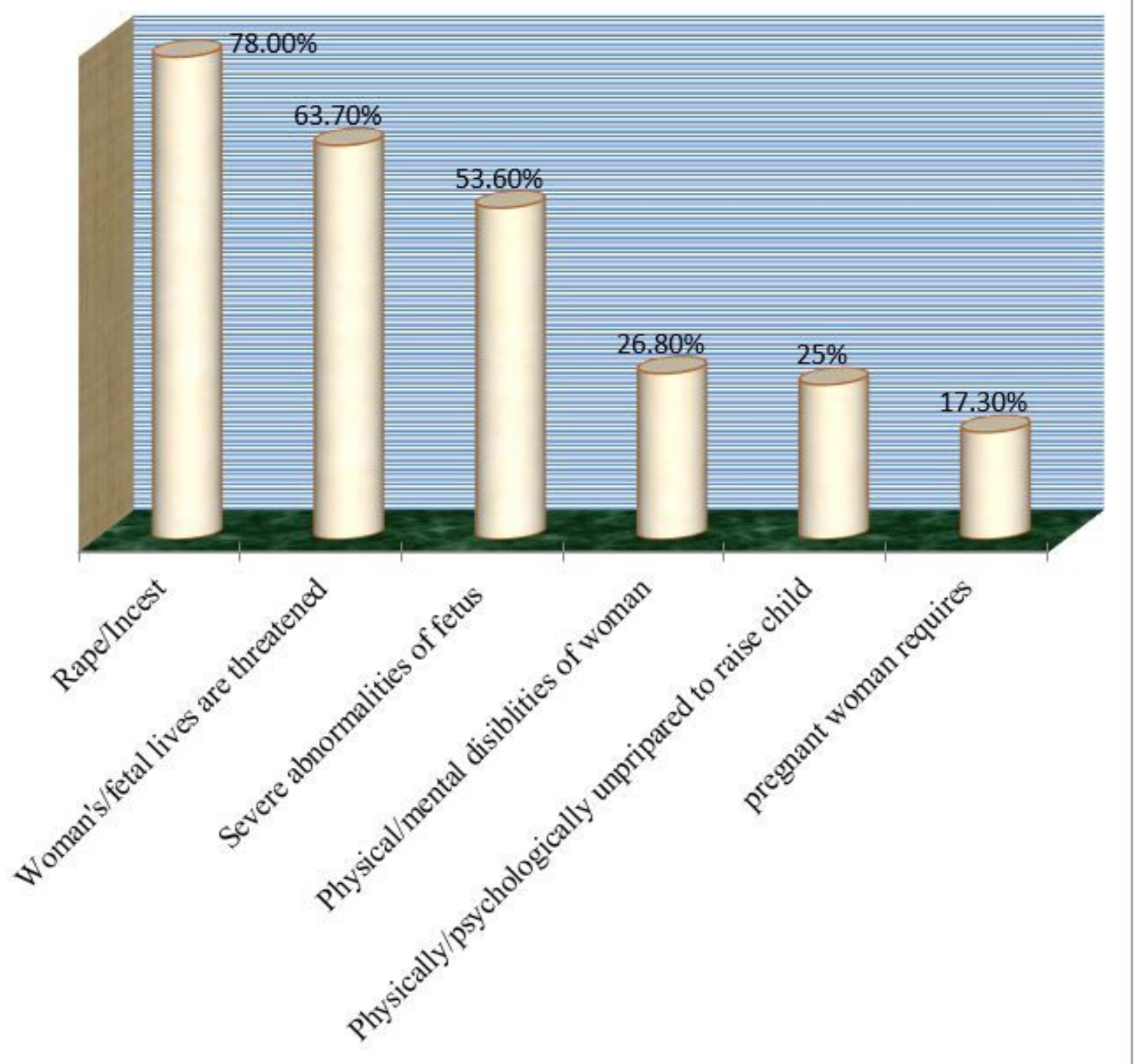

Figure 1

Participant's response on conditions where the Law permits abortion in sexual and reproductive health clinics of Addis Ababa, February 30-March 30, 2018. 


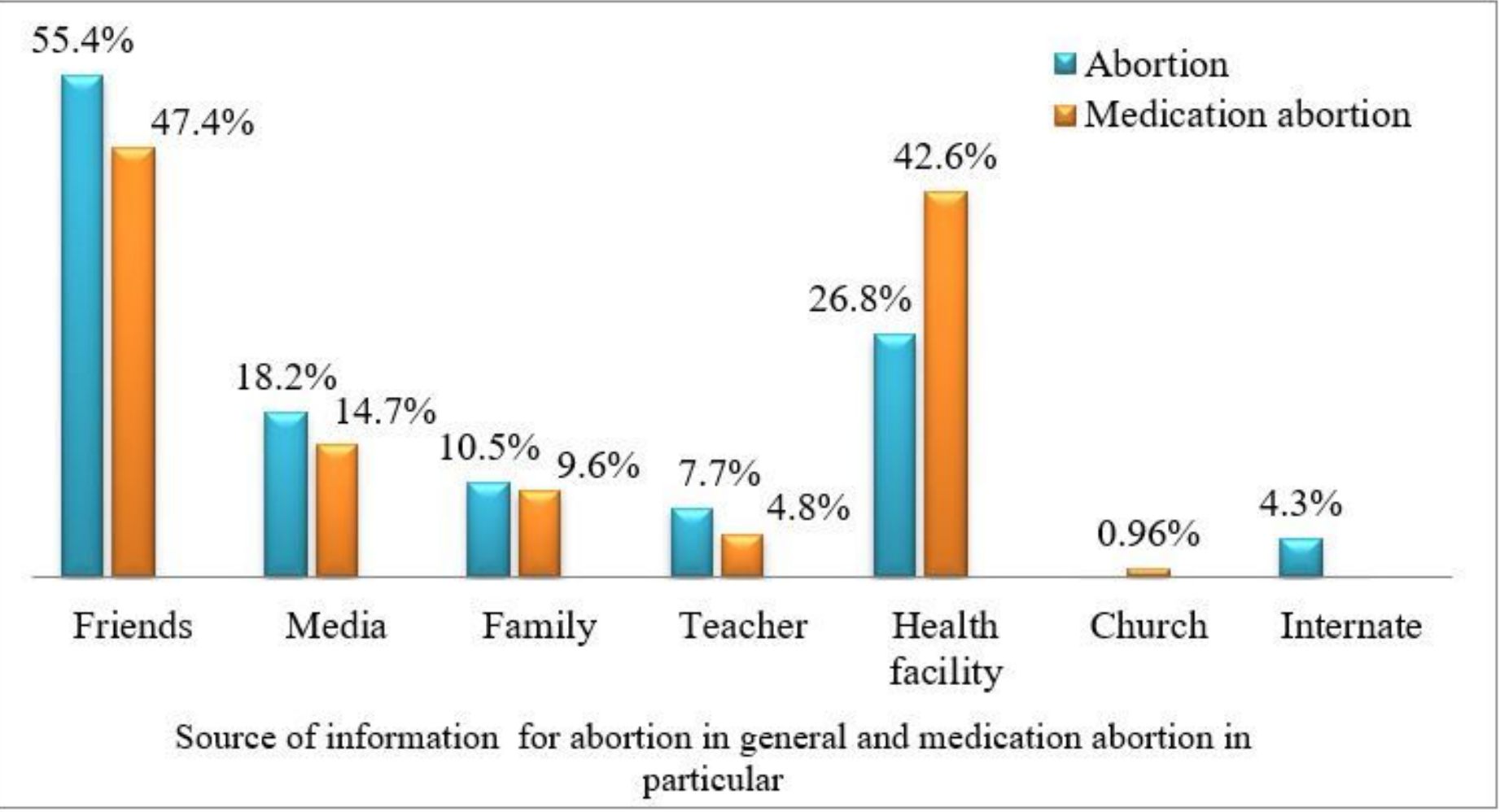

\section{Figure 2}

Major sources of information for abortion in general and medication abortion for reproductive age woman in selected sexual and reproductive health clinics of Addis Ababa, February 30-March 30, 2018.

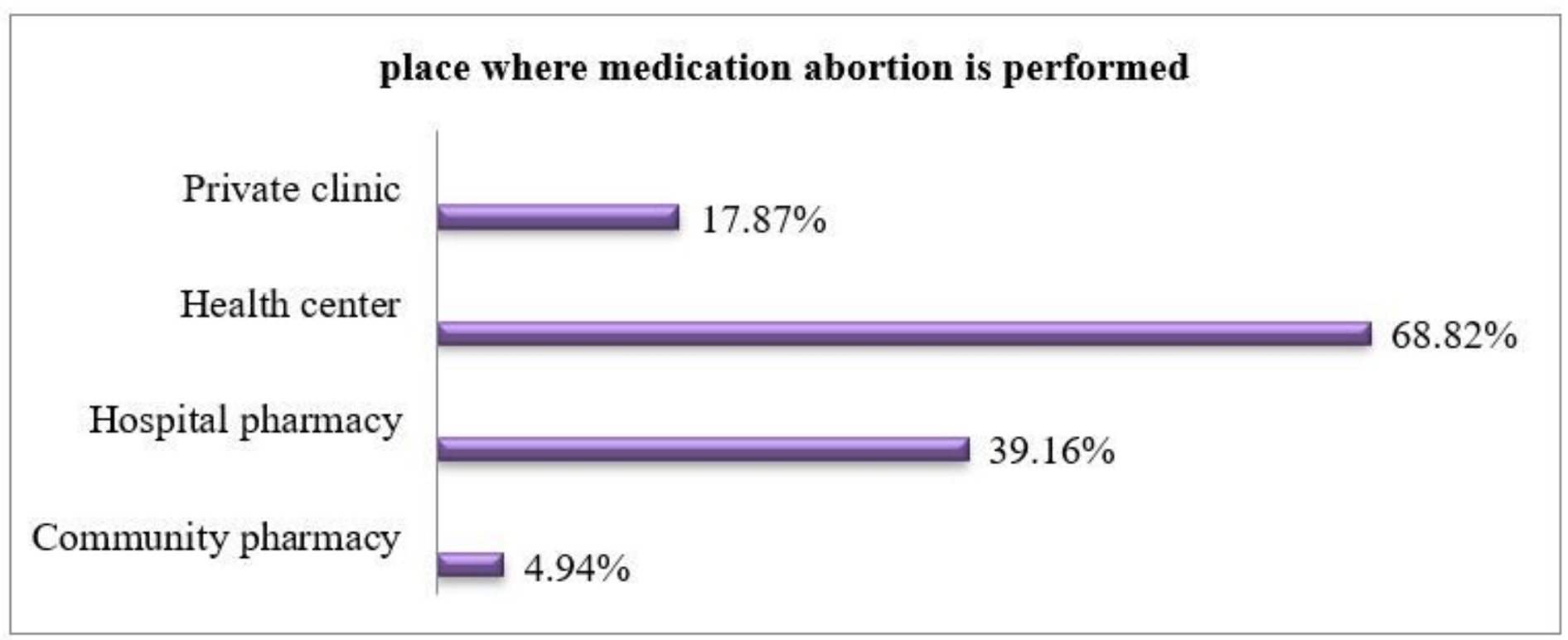

Figure 3

Place where medication abortion is performed, Addis Ababa, February30-March 30, 2018. 


\section{Supplementary Files}

This is a list of supplementary files associated with this preprint. Click to download.

- Formulas.docx

- Table.docx

- Suplmentary.docx 